\title{
Production and Insurance under Regret Aversion*
}

\author{
Kit Pong Wong ${ }^{\dagger}$ \\ University of Hong Kong
}

April 2012

\begin{abstract}
This paper examines the behavior of a regret-averse producer facing revenue risk. To insure against the revenue risk, the producer can purchase a coinsurance contract with an endogenously chosen coinsurance rate. Regret-averse preferences are characterized by a utility function that includes disutility from having chosen ex-post suboptimal alternatives. We show that the regret-averse producer never fully insures against the revenue risk even though the coinsurance contract is actuarially fair. When the producer is sufficiently regret averse and the loss probability is high, we further show that the regret-averse producer chooses not to purchase the actuarially fair coinsurance contract. Even when purchasing the actuarially fair coinsurance contract is optimal, we derive sufficient conditions under which the regret-averse producer reduces the optimal output level as compared to that without the coinsurance contract. These results are distinct from those under pure risk aversion, thereby making the consideration of regret aversion crucial.
\end{abstract}

JEL classification: D21; D24; D81; G22

Keywords: Insurance; Production; Regret theory; Revenue Risk

\footnotetext{
${ }^{*}$ I would like to thank Udo Broll, Stephen Hall (the editor), and an anonymous referee for their helpful comments and suggestions. The usual disclaimer applies.

${ }^{\dagger}$ Correspondence to: Kit Pong Wong, School of Economics and Finance, University of Hong Kong, Pokfulam Road, Hong Kong. Tel.: 852-2859-1044; fax: 852-2548-1152; e-mail: kpwong@econ.hku.hk (K.P. Wong).
} 


\title{
Production and Insurance under Regret Aversion
}

\begin{abstract}
This paper examines the behavior of a regret-averse producer facing revenue risk. To insure against the revenue risk, the producer can purchase a coinsurance contract with an endogenously chosen coinsurance rate. Regret-averse preferences are characterized by a utility function that includes disutility from having chosen ex-post suboptimal alternatives. We show that the regret-averse producer never fully insures against the revenue risk even though the coinsurance contract is actuarially fair. When the producer is sufficiently regret averse and the loss probability is high, we further show that the regret-averse producer chooses not to purchase the actuarially fair coinsurance contract. Even when purchasing the actuarially fair coinsurance contract is optimal, we derive sufficient conditions under which the regret-averse producer reduces the optimal output level as compared to that without the coinsurance contract. These results are distinct from those under pure risk aversion, thereby making the consideration of regret aversion crucial.
\end{abstract}

JEL classification: D21; D24; D81; G22

Keywords: Insurance; Production; Regret theory; Revenue Risk

\section{Introduction}

Revenue insurance, such as multiple peril crop insurance (Mahul and Vermersch, 2000) and livestock revenue insurance (Hart et al., 2001), has been introduced for many agricultural products, which serves as a risk-sharing mechanism between farmers and insurers. The literature that examines the effect of revenue insurance on the behavior of a risk-averse producer is largely conducted within the von Neumann-Morgenstern expected utility context (see, e.g., Machnes, 1995; Ford et al., 1996; Wong, 2000; Machnes and Wong, 2003; Hau, 2006; to name just a few). Such an approach rules out the situation that the producer may have a desire to avoid consequences wherein the producer appears to have made ex-post 
suboptimal decisions, which are de facto optimal ex ante based on the information available at that time. To account for this consideration, Bell $(1982,1983)$ and Loomes and Sugden (1982) propose regret theory that defines regret as the disutility arising from not having chosen the ex-post optimal alternative, which is later axiomatized by Quiggin (1994) and Sugden (1993). Regret theory is supported by a large body of experimental literature that documents regret-averse preferences among individuals (see, e.g., Loomes, 1988; Loomes et al., 1992; Loomes and Sugden, 1987; Starmer and Sugden, 1993).

The purposes of this paper are to incorporate regret theory into the behavior of a producer under uncertainty in general, and examine how regret aversion affects the producer's production and insurance decisions in particular. We model uncertainty as a shock to the producer's revenue, which can be insured against by purchasing a coinsurance contract that the producer can choose a coinsurance rate. Following the seminal work of Braun and Muermann (2004) and Wong (2011), we characterize the producer's regret-averse preferences by a utility function that includes disutility from having chosen ex-post suboptimal alternatives. The extent of regret depends on the difference between the utility level of the actual profit and that of the maximum profit attained by making the optimal production and insurance decisions had the producer learned the true outcome of the revenue risk in advance.

In the absence of insurance against the revenue risk, we show that introducing regret aversion to the producer can induce the producer to produce more or less than the optimal output level that would have been chosen if the producer were purely risk averse. Since the regret-averse producer has to take into account the impact of regret, the optimal output level becomes less extreme as compared to that of the purely risk-averse producer. The global effect of regret aversion on the producer's production decision is therefore to reduce the sensitivity of the optimal output level to changes in the probability distribution of the revenue risk. We further show that the optimal output level is less sensitive to changes in the probability distribution of the revenue risk with an increase in the producer's degree of regret aversion, and becomes completely insensitive in the limiting case when the producer is infinitely regret averse. 
When the regret-averse producer can purchase the coinsurance contract that is actuarially fair, we show that full insurance against the revenue risk is never optimal because the producer would regret to a great extent when no loss actually occurs (see also Braun and Muermann, 2004). We further show two novel results. First, the regret-averse producer may not purchase the actuarially fair coinsurance contract. While risk aversion induces the producer to purchase insurance so as to reduce the variation of his/her profit, regret aversion gives rise to a countervailing incentive that induces the producer to purchase no insurance when the loss probability is high so as to minimize regret. We show that the incentive to opt for no insurance due to regret aversion dominates the incentive to opt for insurance due to risk aversion if the producer is sufficiently regret averse and the loss probability is high. Second, even when purchasing the actuarially fair coinsurance contract is optimal, the regret-averse producer does not necessarily produce more with than without the coinsurance contract. In the presence of insurance against the revenue risk, it is well-known from the literature that the risk-averse producer would like to produce more. Regret aversion, however, creates a countervailing incentive that induces the producer to produce less so as to minimize regret. When the producer is sufficiently regret averse and the loss probability is high, we show that the incentive to lower output due to regret aversion dominates the incentive to raise output due to risk aversion, thereby rendering the output-reducing effect of insurance that exists under regret aversion but not under risk aversion.

The rest of this paper is organized as follows. Section 2 delineates the model of a regretaverse producer facing revenue risk. Section 3 examines the global and marginal effects of regret aversion on the producer's production decision in the absence of insurance against the revenue risk. Section 4 characterizes the optimal production and insurance decisions of the regret-averse producer. The final section concludes.

\section{The model}

Consider a producer who produces a single commodity according to a deterministic 
cost function, $C(Q)$, where $Q \geq 0$ is the output level chosen, $C(0)=C^{\prime}(0)=0$, and $C^{\prime}(Q)>0$ and $C^{\prime \prime}(Q)>0$ for all $Q>0$. The output price is exogenously fixed at $P>0$ per unit. There is a shock such that a fraction, $\gamma \in(0,1]$, of the producer's revenue, $P Q$, is lost with probability $p \in(0,1)$. Given that a loss occurs, the producer's actual revenue is only $(1-\gamma) P Q$. To insure against such revenue risk, the producer can purchase a coinsurance contract with an endogenously chosen coinsurance rate, $\alpha \in[0,1] .{ }^{1}$ Specifically, the producer pays an insurance premium, $(1+m) p \gamma \alpha P Q$, and receives an indemnity, $\gamma \alpha P Q$, in case of a loss, where $m \geq 0$ is the loading factor such that $(1+m) p<1$. $^{2}$ To focus on the pure effect of insurance on the behavior of the producer, we restrict our attention to the case that the coinsurance contract is actuarially fair, i.e., we set $m=0$.

For a given output level, $Q$, and a given coinsurance rate, $\alpha$, the producer's profit, $\Pi_{i}(Q, \alpha)$, is given by

$$
\Pi_{i}(Q, \alpha)= \begin{cases}(1-\gamma) P Q-C(Q)+(1-p) \gamma \alpha P Q & \text { if } i=1 \\ P Q-C(Q)-p \gamma \alpha P Q & \text { if } i=0\end{cases}
$$

where $i=1$ or 0 , indicating whether a loss occurs or not, respectively. The producer purchases no insurance if $\alpha=0$, and opts for full insurance if $\alpha=1$. In the latter fullinsurance case, the producer's profit becomes $\Pi_{i}(Q, 1)=(1-p \gamma) P Q-C(Q)$, which is non-stochastic and thus is unaffected by the revenue risk.

Following Braun and Muermann (2004) and Wong (2011), we define the producer to be regret averse if his/her preferences are represented by the following "modified" utility function that includes some compensation for regret:

$$
V(\Pi)=U(\Pi)-\beta G\left[U\left(\Pi^{\max }\right)-U(\Pi)\right]
$$

where $U(\Pi)$ is a von Neumann-Morgenstern utility function with $U^{\prime}(\Pi)>0$ and $U^{\prime \prime}(\Pi)<0$, $\beta \geq 0$ is a constant regret coefficient, and $G(\cdot)$ is a regret function with $G(0)=0, G^{\prime}(\cdot)>0$,

\footnotetext{
${ }^{1}$ The qualitative results are unaffected if we replace the coinsurance contract by a deductible insurance contract.

${ }^{2}$ If $(1+m) p \geq 1$, the indemnity would not exceed the insurance premium when the loss occurs. In this case, the producer has no incentive to purchase any insurance.
} 
and $G^{\prime \prime}(\cdot)>0$. The regret function, $G(\cdot)$, depends on the difference between the utility levels of the actual profit, $\Pi$, and the maximum profit, $\Pi^{\max }$, that the producer could have earned by making the optimal production and insurance decisions had the producer observed the realized value of the revenue shock. Since $\Pi$ cannot exceed $\Pi^{\max }$, the producer experiences disutility from forgoing the possibility of doing better due to the ignorance of the realized revenue shock. If $\beta=0$, the producer becomes a traditional risk-averse expected utility maximizer. It is evident from Eq. (2) that the producer is always risk averse and is also regret averse when $\beta>0$.

To characterize the regret-averse producer's optimal production and insurance decisions, we have to first derive the maximum profit, $\Pi^{\max }$. Suppose that a loss occurs. Using Eq. (1) with $i=1$, we have

$$
\begin{aligned}
\Pi_{1}^{\max } & =\max _{Q \geq 0, \alpha \in[0,1]}(1-\gamma) P Q-C(Q)+(1-p) \gamma \alpha P Q \\
& =(1-p \gamma) P Q_{1}-C\left(Q_{1}\right)
\end{aligned}
$$

where $Q_{1}$ solves $C^{\prime}\left(Q_{1}\right)=(1-p \gamma) P$. On the other hand, if there is no loss, we use Eq. (1) with $i=0$ to derive

$$
\begin{aligned}
\Pi_{0}^{\max } & =\max _{Q \geq 0, \alpha \in[0,1]} P Q-C(Q)-p \gamma \alpha P Q \\
& =P Q_{0}-C\left(Q_{0}\right)
\end{aligned}
$$

where $Q_{0}$ solves $C^{\prime}\left(Q_{0}\right)=P$. It follows from $C^{\prime \prime}(Q)>0$ that $Q_{1}<Q_{0}$.

The ex-ante decision problem of the regret-averse producer is to choose an output level, $Q$, and a coinsurance rate, $\alpha$, so as to maximize the expected value of his/her regrettheoretical utility function:

$$
\begin{aligned}
\max _{Q \geq 0, \alpha \in[0,1]} & p\left\{U\left[\Pi_{1}(Q, \alpha)-\beta G\left\{U\left(\Pi_{1}^{\max }\right)-U\left[\Pi_{1}(Q, \alpha)\right]\right\}\right\}\right. \\
& +(1-p)\left\{U\left[\Pi_{0}(Q, \alpha)-\beta G\left\{U\left(\Pi_{0}^{\max }\right)-U\left[\Pi_{0}(Q, \alpha)\right]\right\}\right\},\right.
\end{aligned}
$$


where $\Pi_{i}(Q, \alpha)$ is given by Eq. (1) for $i=0$ and 1 , and $\Pi_{1}^{\max }$ and $\Pi_{0}^{\max }$ are given by Eqs. (3) and (4), respectively. The first-order conditions for program (5) are given by

$$
\begin{aligned}
& p\left\{1+\beta G^{\prime}\left\{U\left(\Pi_{1}^{\max }\right)-U\left[\Pi_{1}\left(Q^{*}, \alpha^{*}\right)\right]\right\}\right\} \\
& \times U^{\prime}\left[\Pi_{1}\left(Q^{*}, \alpha^{*}\right)\right]\left[(1-\gamma) P-C^{\prime}\left(Q^{*}\right)+(1-p) \gamma \alpha^{*} P\right] \\
& +(1-p)\left\{1+\beta G^{\prime}\left\{U\left(\Pi_{0}^{\max }\right)-U\left[\Pi_{0}\left(Q^{*}, \alpha^{*}\right)\right]\right\}\right\} \\
& \times U^{\prime}\left[\Pi_{0}\left(Q^{*}, \alpha^{*}\right)\right]\left[P-C^{\prime}\left(Q^{*}\right)-p \gamma \alpha^{*} P\right]=0
\end{aligned}
$$

and

$$
\begin{aligned}
& p\left\{1+\beta G^{\prime}\left\{U\left(\Pi_{1}^{\max }\right)-U\left[\Pi_{1}\left(Q^{*}, \alpha^{*}\right)\right]\right\}\right\} U^{\prime}\left[\Pi_{1}\left(Q^{*}, \alpha^{*}\right)\right](1-p) \gamma P Q^{*} \\
& -(1-p)\left\{1+\beta G^{\prime}\left\{U\left(\Pi_{0}^{\max }\right)-U\left[\Pi_{0}\left(Q^{*}, \alpha^{*}\right)\right]\right\}\right\} U^{\prime}\left[\Pi_{0}\left(Q^{*}, \alpha^{*}\right)\right] p \gamma P Q^{*} \leq 0,
\end{aligned}
$$

where an asterisk $\left(^{*}\right)$ indicates an optimal level, and condition (7) holds with equality if $\alpha^{*}>0$. The second-order conditions for program (5) are satisfied given the assumed properties of $U(\Pi), C(Q)$, and $G(\cdot)$.

\section{Optimal production decision in the absence of insurance}

In this section, we consider the case that the producer is prohibited from insuring against the revenue risk, i.e., $\alpha \equiv 0$. To characterize the regret-averse producer's optimal production decision, we have to first derive the maximum profit, $\Pi^{\max }$. Suppose that a loss occurs. Using Eq. (1) with $i=1$ and $\alpha=0$, we have

$$
\begin{aligned}
\Pi_{2}^{\max } & =\max _{Q \geq 0}(1-\gamma) P Q-C(Q) \\
& =(1-\gamma) P Q_{2}-C\left(Q_{2}\right),
\end{aligned}
$$


where $Q_{2}$ solves $C^{\prime}\left(Q_{2}\right)=(1-\gamma) P$. On the other hand, if there is no loss, the maximum profit, $\Pi_{0}^{\max }$, is given by Eq. (4) since the producer purchases no insurance even when the coinsurance contract is available. It follows from $C^{\prime \prime}(Q)>0$ that $Q_{2}<Q_{1}<Q_{0}$.

The ex-ante decision problem of the regret-averse producer is to choose an output level, $Q$, to maximize the expected value of his/her regret-theoretical utility function:

$$
\begin{array}{rl}
\max _{Q \geq 0} & p\left\{U\left[\Pi_{1}(Q, 0)\right]-\beta G\left\{U\left(\Pi_{2}^{\max }\right)-U\left[\Pi_{1}(Q, 0)\right]\right\}\right\} \\
& +(1-p)\left\{U\left[\Pi_{0}(Q, 0)\right]-\beta G\left\{U\left(\Pi_{0}^{\max }\right)-U\left[\Pi_{0}(Q, 0)\right]\right\}\right\},
\end{array}
$$

where $\Pi_{i}(Q, 0)$ is given by Eq. (1) for $i=0$ and 1 with $\alpha=0$, and $\Pi_{2}^{\max }$ and $\Pi_{0}^{\max }$ are given by Eqs. (4) and (8), respectively. The first-order condition for program (9) is given by

$$
\begin{aligned}
& p\left\{1+\beta G^{\prime}\left\{U\left(\Pi_{2}^{\max }\right)-U\left[\Pi_{1}\left(Q^{\circ}, 0\right)\right]\right\}\right\} U^{\prime}\left[\Pi_{1}\left(Q^{\circ}, 0\right)\right]\left[(1-\gamma) P-C^{\prime}\left(Q^{\circ}\right)\right] \\
& +(1-p)\left\{1+\beta G^{\prime}\left\{U\left(\Pi_{0}^{\max }\right)-U\left[\Pi_{0}\left(Q^{\circ}, 0\right)\right]\right\}\right\} U^{\prime}\left[\Pi_{0}\left(Q^{\circ}, 0\right)\right]\left[P-C^{\prime}\left(Q^{\circ}\right)\right]=0
\end{aligned}
$$

where $Q^{\circ}$ is the optimal output level. It is evident from Eq. (10) that $(1-\gamma) P<C^{\prime}\left(Q^{\circ}\right)<$ $P$. Since $C^{\prime \prime}(Q)>0$, it follows that $Q_{2}<Q^{\circ}<Q_{0}$.

To examine the global effect of regret aversion on the behavior of the producer, we derive the optimal output level should the producer be purely risk averse, i.e., $\beta=0$. In this case, the first-order condition, Eq. (10), reduces to

$$
p U^{\prime}\left[\Pi_{1}\left(Q^{\dagger}, 0\right)\right]\left[(1-\gamma) P-C^{\prime}\left(Q^{\dagger}\right)\right]+(1-p) U^{\prime}\left[\Pi_{0}\left(Q^{\dagger}, 0\right)\right]\left[P-C^{\prime}\left(Q^{\dagger}\right)\right]=0
$$

where $Q^{\dagger}$ is the purely risk-averse producer's optimal output level. Using Eqs. (10) and (11), we compare $Q^{\circ}$ with $Q^{\dagger}$ to establish the following proposition. All proofs of propositions are relegated to Appendix A. 
Proposition 1. There exists a critical value, $p^{\circ} \in(0,1)$, of the loss probability, $p$, given by

$$
p^{\circ}=\frac{U^{\prime}\left[\Pi_{0}(\bar{Q}, 0)\right]\left[P-C^{\prime}(\bar{Q})\right]}{U^{\prime}\left[\Pi_{0}(\bar{Q}, 0)\right]\left[P-C^{\prime}(\bar{Q})\right]-U^{\prime}\left[\Pi_{1}(\bar{Q}, 0)\right]\left[(1-\gamma) P-C^{\prime}(\bar{Q})\right]},
$$

where $\bar{Q}$ is the output level that solves

$$
U\left(\Pi_{0}^{\max }\right)-U\left[\Pi_{0}(\bar{Q}, 0)\right]=U\left(\Pi_{2}^{\max }\right)-U\left[\Pi_{1}(\bar{Q}, 0)\right]
$$

In the absence of insurance against the revenue risk, if $p<(>) p^{\circ}$, the regret-averse producer optimally chooses a lower (higher) output level than the purely risk-averse producer, i.e., $Q^{\circ}<(>) Q^{\dagger}$. If $p=p^{\circ}$, then $Q^{\circ}=Q^{\dagger}=\bar{Q}$.

Eq. (13) defines the output level, $\bar{Q}$, at which the extent of regret in the no-loss state, $U\left(\Pi_{0}^{\max }\right)-U\left[\Pi_{0}(\bar{Q}, 0)\right]$, is equal to that in the loss state, $U\left(\Pi_{2}^{\max }\right)-U\left[\Pi_{1}(\bar{Q}, 0)\right]$. To see the intuition underlying Proposition 1, suppose that the producer is purely risk averse. When the loss probability, $p$, is small, the producer optimally chooses the output level, $Q^{\dagger}$, closer to the optimal output level without a loss, $Q_{0}$, and further way from the one with a loss, $Q_{2}$, so as to minimize the variability of his/her profit. Introducing regret aversion to the producer makes the producer take into account the substantial disutility from the large discrepancy of the output level, $Q^{\dagger}-Q_{2}$, when a loss occurs. To avoid regret, the regret-averse producer optimally adjusts the output level downward from $Q^{\dagger}$ to move a bit closer to $Q_{2}$ so that $Q^{\circ}<Q^{\dagger}$. On the other hand, in the case that $p$ is large, $Q^{\dagger}$ is closer to $Q_{2}$ and further away from $Q_{0}$. The regret-averse producer as such optimally adjusts the output level upward from $Q^{\dagger}$ to reduce the discrepancy of the output level, $Q_{0}-Q^{\circ}$, when a loss does not occur. Hence, we have $Q^{\circ}>Q^{\dagger}$. $^{3}$

An immediate implication of Proposition 1 is that the production decision of the regretaverse producer is less sensitive to changes in the probability distribution of the revenue risk than that of the purely risk-averse producer. The following proposition shows that

\footnotetext{
${ }^{3}$ Wong (2011) derives similar results in the context of a regret-averse banking firm.
} 
this result extends to a marginal increase in the degree of regret aversion as gauged by the constant regret coefficient, $\beta$.

Proposition 2. In the absence of insurance against the revenue risk, the regret-averse producer's optimal output level, $Q^{\circ}$, increases (decreases) with an increase in the constant regret coefficient, $\beta$, i.e., $\mathrm{d} Q^{\circ} / \mathrm{d} \beta>(<) 0$, if the loss probability, $p$, is smaller (larger) than the critical value, $p^{\circ}$.

As the producer becomes more regret averse, the sensitivity of his/her optimal output level to changes in the probability distribution of the revenue risk goes down. In the limit when the producer is infinitely regret averse, the optimal output level, $\bar{Q}$, is the one that solves Eq. (13), rendering the compensation for regret to be independent of the revenue risk. It is evident from Eq. (13) that the infinitely regret-averse producer's optimal output level, $\bar{Q}$, is indeed completely insensitive to changes in the probability distribution of the revenue risk.

\section{Optimal production and insurance decisions}

In this section, we resume the original case that the producer can insure against the revenue risk. As a benchmark, we first consider that the producer is purely risk averse, i.e., $\beta=0$. Solving Eq. (6) and condition (7) with $\beta=0$ yields the following proposition.

Proposition 3. The purely risk-averse producer uses the actuarially fair coinsurance contract to fully insure against the revenue risk. The producer's optimal output level, $Q_{1}$, solves $C^{\prime}\left(Q_{1}\right)=(1-p \gamma)$, which is larger than the one without insurance, $Q^{\dagger}$, for all $p \in(0,1)$.

Since the coinsurance contract is actuarially fair, the purely risk-averse producer optimally opts for full insurance. The producer's profit becomes $\Pi_{i}(Q, 1)=(1-p \gamma) P Q-C(Q)$, 
which is non-stochastic. Hence, the producer optimally produces at the output level, $Q_{1}$, that solves $C^{\prime}\left(Q_{1}\right)=(1-p \gamma) P$, which is also the optimal output level should the producer be risk neutral. By fully insuring against the revenue risk, the risk-averse producer is induced to produce more so that $Q_{1}$ exceeds the output level without insurance, $Q^{\dagger}$ (see, e.g., Machnes, 1995; Ford et al., 1996; Wong, 2000; Machnes and Wong, 2003; Hau, 2006).

To examine the production and insurance decisions of the regret-averse producer, we define the following function:

$$
\begin{aligned}
H(p)= & \left\{1+\beta G^{\prime}\left\{U\left(\Pi_{1}^{\max }\right)-U\left[\Pi_{1}\left(Q^{\circ}, 0\right)\right]\right\}\right\} U^{\prime}\left[\Pi_{1}\left(Q^{\circ}, 0\right)\right] \\
& -\left\{1+\beta G^{\prime}\left\{U\left(\Pi_{0}^{\max }\right)-U\left[\Pi_{0}\left(Q^{\circ}, 0\right)\right]\right\}\right\} U^{\prime}\left[\Pi_{0}\left(Q^{\circ}, 0\right)\right]
\end{aligned}
$$

where $Q^{\circ}$ is the optimal output level in the absence of insurance against the revenue risk. Solving Eq. (6) and condition (7) yields the following proposition.

Proposition 4. Given that the following condition holds:

$$
\frac{1+\beta G^{\prime}(0)}{1+\beta G^{\prime}\left\{U\left(\Pi_{0}^{\max }\right)-U\left[\Pi_{0}\left(Q_{2}, 0\right)\right]\right\}}<\frac{U^{\prime}\left[\Pi_{0}\left(Q_{2}, 0\right)\right]}{U^{\prime}\left[\Pi_{1}\left(Q_{2}, 0\right)\right]}
$$

there exists a critical value, $p^{*} \in(0,1)$, of the loss probability, $p$, at which $H\left(p^{*}\right)=0$. For all $p \in\left(0, p^{*}\right)$, the regret-averse producer chooses the optimal output level, $Q_{1}$, that solves $C^{\prime}\left(Q_{1}\right)=(1-p \gamma) P$, and uses the actuarially fair coinsurance contract to partially insure against the revenue risk such that the optimal coinsurance rate, $\alpha^{*} \in(0,1)$, solves

$$
\begin{aligned}
& \left\{1+\beta G^{\prime}\left\{U\left(\Pi_{1}^{\max }\right)-U\left[\Pi_{1}\left(Q_{1}, \alpha^{*}\right)\right]\right\}\right\} U^{\prime}\left[\Pi_{1}\left(Q_{1}, \alpha^{*}\right)\right] \\
& -\left\{1+\beta G^{\prime}\left\{U\left(\Pi_{0}^{\max }\right)-U\left[\Pi_{0}\left(Q_{1}, \alpha^{*}\right)\right]\right\}\right\} U^{\prime}\left[\Pi_{0}\left(Q_{1}, \alpha^{*}\right)\right]=0 .
\end{aligned}
$$

For all $p \in\left[p^{*}, 1\right)$, the producer purchases no insurance and chooses the optimal output level, $Q^{\circ}$, that solves Eq. (10). If condition (15) does not hold, the producer's optimal coinsurance rate, $\alpha^{*} \in(0,1)$, solves $E q$. (16) and the optimal output level is $Q_{1}$ for all $p \in(0,1)$. 
To see the intuition for Proposition 4, we write Eq. (1) as

$$
\Pi_{i}(Q, \alpha)= \begin{cases}(1-p \gamma) P Q-C(Q)-(1-p)(1-\alpha) \gamma P Q & \text { if } i=1, \\ (1-p \gamma) P Q-C(Q)+p(1-\alpha) \gamma P Q & \text { if } i=0 .\end{cases}
$$

As is evident from Eq. (17), the producer's production decision affects his/her exposure to the revenue risk through the last term on the right-hand side of Eq. (17). This term could have been completely eliminated had the producer chosen full insurance, i.e., $\alpha=1$, within his/her own discretion. As such, the revenue risk should not affect the producer's production decision as long as the producer finds it optimal to insure against the revenue risk. The optimal output level is then solely determined by maximizing $(1-p \gamma) P Q-C(Q)$, thereby giving rise to the optimality condition, $C^{\prime}\left(Q_{1}\right)=(1-p \gamma) P$.

Proposition 4 shows that the regret-averse producer never opts for full insurance against the revenue risk even though the coinsurance contract is actuarially fair, which is consistent with the findings of Braun and Muermann (2004). To see the intuition, suppose that the regret-averse producer fully insures against the revenue risk. While the producer's profit is non-stochastic under full insurance, the extent of regret is not. Specifically, there is no regret only when a loss occurs, and a lot of regret otherwise. Since the regret function, $G(\cdot)$, is convex, the regret-averse producer is induced to lower the coinsurance rate below unity so as to reduce the variation of regret between the loss and no-loss states. Such incentive, however, is absent for the purely risk-averse producer, thereby making the results of Propositions 3 and 4 distinct from each other.

A novel result of Proposition 4 is that there are reasonable conditions under which the regret-averse producer has no incentive to purchase the actuarially fair coinsurance contract. Suppose that

$$
\frac{G^{\prime}(0)}{G^{\prime}\left\{U\left(\Pi_{0}^{\max }\right)-U\left[\Pi_{0}\left(Q_{2}, 0\right)\right]\right\}}<\frac{U^{\prime}\left[\Pi_{0}\left(Q_{2}, 0\right)\right]}{U^{\prime}\left[\Pi_{1}\left(Q_{2}, 0\right)\right]}
$$

which holds if $G(\cdot)$ is sufficiently convex in general, and if $G^{\prime}(0)=0$ in particular. In this 
case, we can write condition (15) as

$$
\beta>\frac{U^{\prime}\left[\Pi_{1}\left(Q_{2}, 0\right)\right]-U^{\prime}\left[\Pi_{0}\left(Q_{2}, 0\right)\right]}{G^{\prime}\left\{U\left(\Pi_{0}^{\max }\right)-U\left[\Pi_{0}\left(Q_{2}, 0\right)\right]\right\} U^{\prime}\left[\Pi_{0}\left(Q_{2}, 0\right)\right]-G^{\prime}(0) U^{\prime}\left[\Pi_{1}\left(Q_{2}, 0\right)\right]},
$$

where the expression on the right-hand side of condition (19) is positive given condition (18). Hence, if the producer is sufficiently regret averse such that condition (19) holds, it follows from Proposition 4 that purchasing the actuarially fair coinsurance contract is no longer optimal when the loss probability, $p$, is high, i.e., $p \in\left[p^{*}, 1\right)$, where $p^{*} \in(0,1)$ is the critical loss probability that solves $H\left(p^{*}\right)=0{ }^{4}$

To see the underlying intuition, suppose that the regret-averse producer purchases no insurance against the revenue risk. As such, the producer's profit in the no-loss state is higher than that in the loss state. Risk aversion induces the producer to purchase insurance so as to reduce the variation of his/her profit between the two states. However, there is a countervailing incentive that comes from regret aversion. Under no insurance, the extent of regret in the loss state is given by $U\left(\Pi_{1}^{\max }\right)-U\left[\Pi_{1}\left(Q^{\circ}, 0\right)\right]$, while that in the no-loss state is given by $U\left(\Pi_{0}^{\max }\right)-U\left[\Pi_{0}\left(Q^{\circ}, 0\right)\right]$. Since both $Q^{\circ}$ and $Q_{1}$ approach $Q_{2}$ as the loss probability, $p$, goes to unity, there is no regret in the loss state and the extent of regret in the no-loss state is minimized in this extreme case wherein the producer does not insure against the revenue risk. Hence, when the producer is sufficiently regret averse and the loss probability is high, the incentive to opt for no insurance due to regret aversion dominates the incentive to opt for insurance due to risk aversion. The regret-averse producer as such optimally refrains from insuring against the revenue risk.

Define the following function:

$$
\begin{aligned}
K(p)= & \left\{1+\beta G^{\prime}\left\{U\left(\Pi_{2}^{\max }\right)-U\left[\Pi_{1}\left(Q^{\circ}, 0\right)\right]\right\}\right\} U^{\prime}\left[\Pi_{1}\left(Q^{\circ}, 0\right)\right] \\
& -\left\{1+\beta G^{\prime}\left\{U\left(\Pi_{0}^{\max }\right)-U\left[\Pi_{0}\left(Q^{\circ}, 0\right)\right]\right\}\right\} U^{\prime}\left[\Pi_{0}\left(Q^{\circ}, 0\right)\right],
\end{aligned}
$$

\footnotetext{
${ }^{4}$ In Braun and Muermann (2004), the loss is exogenously determined, which is tantamount to fixing the output level in our model. In this case, $U\left(\Pi_{0}^{\max }\right)=U\left[\Pi_{0}\left(Q_{2}, 0\right)\right]$ since $Q_{0}=Q_{2}$. Condition (15) as such never holds, thereby making the purchase of the actuarially fair coinsurance contract always optimal in the context of Braun and Muermann (2004).
} 
where $Q^{\circ}$ is the optimal output level in the absence of insurance against the revenue risk. Since $\Pi_{1}^{\max }>\Pi_{2}^{\max }$ and $G^{\prime \prime}(\cdot)>0$, it follows from Eqs. (14) and (20) that $H(p)>K(p)$ for all $p \in(0,1)$. We show in the following proposition that the regret-averse producer who optimally purchases the actuarially fair coinsurance contract does not necessarily produce more than $Q^{\circ}$, in sharp contrast to what Proposition 3 would have suggested.

Proposition 5. Given that condition (15) holds, there exists a critical value, $p^{* *} \in\left(0, p^{*}\right)$, of the loss probability, $p$, at which $K\left(p^{* *}\right)=0$. For all $p \in\left(0, p^{*}\right)$, the regret-averse producer purchases the actuarially fair coinsurance contract and produces at the optimal output level, $Q_{1}$, that is larger than, equal to, or smaller than the one without insurance, $Q^{\circ}$, depending on whether $p \in\left(0, p^{* *}\right), p=p^{* *}$, or $p \in\left(p^{* *}, p^{*}\right)$, respectively. If condition (15) does not hold, the producer always produces more with than without insurance, i.e., $Q_{1}>Q^{\circ}$, for all $p \in(0,1)$.

The intuition for Proposition 5 is as follows. In the absence of insurance against the revenue risk, the producer's profit in the no-loss state is higher than that in the loss state. The extent of regret in the loss state is given by $U\left(\Pi_{2}^{\max }\right)-U\left[\Pi_{1}\left(Q^{\circ}, 0\right)\right]$, while that in the no-loss state is given by $U\left(\Pi_{0}^{\max }\right)-U\left[\Pi_{0}\left(Q^{\circ}, 0\right)\right]$. If the producer is allowed to purchase the actuarially fair coinsurance contract, risk aversion induces the producer to produce more than $Q^{\circ}$. Regret aversion, on the other hand, creates a countervailing incentive that induces the producer to produce less. To see this, suppose that the loss probability, $p$, is sufficiently close to $p^{*}$ so that the optimal coinsurance rate, $\alpha^{*}$, is very close to zero (see Proposition $4)$. If the producer still produces at $Q^{\circ}$ in the presence of insurance, the extent of regret in the no-loss state remains unchanged but that in the loss state increases since $\Pi_{1}^{\max }>\Pi_{2}^{\max }$. Given that the regret function, $G(\cdot)$, is convex, the regret-averse producer has incentive to produce less than $Q^{\circ}$ so as to reduce the variation of regret between the two states. Hence, when the producer is sufficiently regret averse and the loss probability is high, the incentive to lower output due to regret aversion dominates the incentive to raise output due to risk aversion. The regret-averse producer as such optimally produces less with than without the 
actuarially fair coinsurance contract.

\section{Conclusion}

This paper examines the behavior of a regret-averse producer facing revenue risk. To insure against the revenue risk, the producer can purchase a coinsurance contract with an endogenously chosen coinsurance rate. Following the regret-theoretical approach of Braun and Muermann (2004) and Wong (2011), we characterize the producer's regret-averse preferences by a utility function that includes disutility from having chosen ex-post suboptimal alternatives. We show that the regret-averse producer never opts for full insurance against the revenue risk even though the coinsurance contract is actuarially fair. When the producer is sufficiently regret averse and the loss probability is high, we further show that the regret-averse producer dose not purchase the actuarially fair coinsurance contract. Even when purchasing the actuarially fair coinsurance contract is optimal, the regret-averse producer does not necessarily produce more with than without the coinsurance contract. These results are in sharp contrast to those under pure risk aversion, suggesting that regret aversion plays a distinctive role in shaping the optimal production and insurance decisions in the presence of revenue risk.

\section{Appendix A}

Proof of Proposition 1. The second-order condition for program (9) is given by

$$
\begin{aligned}
S O C^{\circ}= & -p\left\{1+\beta G^{\prime}\left\{U\left(\Pi_{2}^{\max }\right)-U\left[\Pi_{1}\left(Q^{\circ}, 0\right)\right]\right\}\right\} U^{\prime}\left[\Pi_{1}\left(Q^{\circ}, 0\right)\right] C^{\prime \prime}\left(Q^{\circ}\right) \\
& -(1-p)\left\{1+\beta G^{\prime}\left\{U\left(\Pi_{0}^{\max }\right)-U\left[\Pi_{0}\left(Q^{\circ}, 0\right)\right]\right\}\right\} U^{\prime}\left[\Pi_{0}\left(Q^{\circ}, 0\right)\right] C^{\prime \prime}\left(Q^{\circ}\right) \\
& +p\left\{1+\beta G^{\prime}\left\{U\left(\Pi_{2}^{\max }\right)-U\left[\Pi_{1}\left(Q^{\circ}, 0\right)\right]\right\}\right\} U^{\prime \prime}\left[\Pi_{1}\left(Q^{\circ}, 0\right)\right]\left[(1-\gamma) P-C^{\prime}\left(Q^{\circ}\right)\right]^{2}
\end{aligned}
$$




$$
\begin{aligned}
& +(1-p)\left\{1+\beta G^{\prime}\left\{U\left(\Pi_{0}^{\max }\right)-U\left[\Pi_{0}\left(Q^{\circ}, 0\right)\right]\right\}\right\} U^{\prime \prime}\left[\Pi_{0}\left(Q^{\circ}, 0\right)\right]\left[P-C^{\prime}\left(Q^{\circ}\right)\right]^{2} \\
& -p \beta G^{\prime \prime}\left\{U\left(\Pi_{1}^{\max }\right)-U\left[\Pi_{1}\left(Q^{\circ}, 0\right)\right]\right\} U^{\prime}\left[\Pi_{1}\left(Q^{\circ}, 0\right)\right]^{2}\left[(1-\gamma) P-C^{\prime}\left(Q^{\circ}\right)\right]^{2} \\
& -(1-p) \beta G^{\prime \prime}\left\{U\left(\Pi_{0}^{\max }\right)-U\left[\Pi_{0}\left(Q^{\circ}, 0\right)\right]\right\} U^{\prime}\left[\Pi_{0}\left(Q^{\circ}, 0\right)\right]^{2}\left[P-C^{\prime}\left(Q^{\circ}\right)\right]^{2} \\
& <0
\end{aligned}
$$

which is satisfied given $U^{\prime \prime}(\Pi)<0, C^{\prime \prime}(Q)>0, G^{\prime}(\cdot)>0$, and $G^{\prime \prime}(\cdot)>0$. Hence, $Q^{\circ}$ that solves Eq. (10) is the unique maximum solution. Similarly, when $\beta=0$, the second-order condition is given by $S O C^{\dagger}<0$, which is given by Eq. (A.1) with $\beta=0$ and $Q^{\circ}$ replaced by $Q^{\dagger}$. Hence, $Q^{\dagger}$ that solves Eq. (11) is the unique maximum solution in the case of pure risk aversion.

Evaluating the left-hand side of Eq. (10) at $Q^{\circ}=Q^{\dagger}$ yields

$$
\begin{aligned}
& \beta(1-p) U^{\prime}\left[\Pi_{1}\left(Q^{\dagger}, 0\right)\right]\left[P-C^{\prime}\left(Q^{\dagger}\right)\right] \\
& \times\left\{G^{\prime}\left\{U\left(\Pi_{0}^{\max }\right)-U\left[\Pi_{0}\left(Q^{\dagger}, 0\right)\right]\right\}-G^{\prime}\left\{U\left(\Pi_{2}^{\max }\right)-U\left[\Pi_{1}\left(Q^{\dagger}, 0\right)\right]\right\}\right\},
\end{aligned}
$$

where we have used Eq. (11). Define the following function:

$$
F(p)=U\left(\Pi_{0}^{\max }\right)-U\left[\Pi_{0}\left(Q^{\dagger}, 0\right)\right]-\left\{U\left(\Pi_{2}^{\max }\right)-U\left[\Pi_{1}\left(Q^{\dagger}, 0\right)\right]\right\}
$$

Since $G^{\prime \prime}(\cdot)>0$, expression (A.2) is positive or negative, depending on whether $F(p)$ is positive or negative, respectively. Differentiating Eq. (A.3) with respect to $p$ yields

$$
F^{\prime}(p)=\left\{U^{\prime}\left[\Pi_{1}\left(Q^{\dagger}, 0\right)\right]\left[(1-\gamma) P-C^{\prime}\left(Q^{\dagger}\right)\right]-U^{\prime}\left[\Pi_{0}\left(Q^{\dagger}, 0\right)\right]\left[P-C^{\prime}\left(Q^{\dagger}\right)\right]\right\} \frac{\mathrm{d} Q^{\dagger}}{\mathrm{d} p}
$$

From Eq. (11), we have $(1-\gamma) P<C^{\prime}\left(Q^{\dagger}\right)<P$. Eq. (A.4) then implies that $F^{\prime}(p)$ has the opposite sign to that of $\mathrm{d} Q^{\dagger} / \mathrm{d} p$. Totally differentiating Eq. (11) with respect to $p$ yields

$$
\frac{\mathrm{d} Q^{\dagger}}{\mathrm{d} p}=-\frac{1}{S O C^{\dagger}}\left\{U^{\prime}\left[\Pi_{1}\left(Q^{\dagger}, 0\right)\right]\left[(1-\gamma) P-C^{\prime}\left(Q^{\dagger}\right)\right]-U^{\prime}\left[\Pi_{0}\left(Q^{\dagger}, 0\right)\right]\left[P-C^{\prime}\left(Q^{\dagger}\right)\right]\right\}
$$




$$
=\frac{1}{p S O C^{\dagger}} U^{\prime}\left[\Pi_{0}\left(Q^{\dagger}, 0\right)\right]\left[P-C^{\prime}\left(Q^{\dagger}\right)\right]<0,
$$

where the second equality follows from Eq. (11), and the inequality follows from $S O C^{\dagger}<0$ and $C^{\prime}\left(Q^{\dagger}\right)<P$. Hence, Eqs. (A.4) and (A.5) imply that $F^{\prime}(p)>0$.

When $p$ approaches zero, we have $Q^{\dagger}$ approaches $Q_{0}$. Since $\Pi_{2}^{\max }>\Pi_{1}\left(Q_{0}, 0\right)$, Eq. (A.3) implies that $F(0)=U\left[\Pi_{1}\left(Q_{0}, 0\right)\right]-U\left(\Pi_{2}^{\max }\right)<0$. On the other hand, when $p$ approaches one, we have $Q^{\dagger}$ approaches $Q_{2}$. Since $\Pi_{0}^{\max }>\Pi_{0}\left(Q_{2}, 0\right)$, Eq. (A.3) implies that $F(1)=U\left(\Pi_{0}^{\max }\right)-U\left[\Pi_{0}\left(Q_{2}, 0\right)\right]>0$. Hence, there must exist a unique point, $p^{\circ} \in(0,1)$, that solves $F\left(p^{\circ}\right)=0$. Solving $F\left(p^{\circ}\right)=0$ for $\bar{Q}$ yields Eq. (13). Fixing $Q^{\dagger}=\bar{Q}$ in Eq. (11) and solving for $p^{\circ}$ yields Eq. (12).

For all $p \in\left(0, p^{\circ}\right)$, we have $F(p)<0$ and thus expression (A.2) is negative. On the other hand, for all $p \in\left(p^{\circ}, 1\right)$, we have $F(p)>0$ and thus expression (A.2) is positive. It then follows from Eqs. (10) and (A.1) that $\bar{Q}<Q^{\circ}<Q^{\dagger}$ for all $p \in\left(0, p^{\circ}\right)$ and that $\bar{Q}>Q^{\circ}>Q^{\dagger}$ for all $p \in\left(p^{\circ}, 1\right)$. If $p=p^{\circ}$, then $Q^{\circ}=Q^{\dagger}=\bar{Q}$.

Proof of Proposition 2. Totally differentiating Eq. (10) with respect to $\beta$ yields

$$
\begin{aligned}
& \frac{\mathrm{d} Q^{\circ}}{\mathrm{d} \beta}=-\frac{1}{S O C^{\circ}}\left\{p G^{\prime}\left\{U\left(\Pi_{1}^{\max }\right)-U\left[\Pi_{1}\left(Q^{\circ}, 0\right)\right]\right\} U^{\prime}\left[\Pi_{1}\left(Q^{\circ}, 0\right)\right]\left[(1-\gamma) P-C^{\prime}\left(Q^{\circ}\right)\right]\right. \\
& \left.+(1-p) G^{\prime}\left\{U\left(\Pi_{0}^{\max }\right)-U\left[\Pi_{0}\left(Q^{\circ}, 0\right)\right]\right\} U^{\prime}\left[\Pi_{0}\left(Q^{\circ}, 0\right)\right]\left[P-C^{\prime}\left(Q^{\circ}\right)\right]\right\} \\
& =\frac{1}{\beta S O C^{\circ}}\left\{p U^{\prime}\left[\Pi_{1}\left(Q^{\circ}\right)\right]\left[(1-\gamma) P-C^{\prime}\left(Q^{\circ}\right)\right]+(1-p) U^{\prime}\left[\Pi_{0}\left(Q^{\circ}\right)\right]\left[P-C^{\prime}\left(Q^{\circ}\right)\right]\right\},
\end{aligned}
$$

where the second equality follows from Eq. (10). From Proposition 1, we know that $Q^{\circ}<Q^{\dagger}$ for all $p \in\left(0, p^{\circ}\right)$. Hence, it follows from Eqs. (11) and $S O C^{\dagger}<0$ that the expression inside the curly brackets on the right-hand side of Eq. (A.6) is negative. Thus, Eqs. (A.1) and (A.6) imply that $\mathrm{d} Q^{\circ} / \mathrm{d} \beta>0$ for all $p \in\left(0, p^{\circ}\right)$. On the other hand, for all $p \in\left(p^{\circ}, 1\right)$ we have $Q^{\circ}>Q^{\dagger}$ from Proposition 1. Hence, it follows from Eq. (11) and $S O C^{\dagger}<0$ that the expression inside the curly brackets on the right-hand side of Eq. (A.6) is positive. Thus, Eqs. (A.1) and (A.6) imply that $\mathrm{d} Q^{\circ} / \mathrm{d} \beta<0$ for all $p \in\left(p^{\circ}, 1\right)$. If $p=p^{\circ}$, then $\mathrm{d} \bar{Q} / \mathrm{d} \beta=0$. 
Proof of Proposition 3. Condition (7) with $\beta=0$ can be written as $U^{\prime}\left[\Pi_{1}\left(Q^{*}, \alpha^{*}\right)\right]-$ $U^{\prime}\left[\Pi_{0}\left(Q^{*}, \alpha^{*}\right)\right] \leq 0$. Since $\Pi_{0}\left(Q^{*}, 0\right)-\Pi_{1}\left(Q^{*}, 0\right)=\gamma P Q^{*}>0$, risk aversion implies that $U^{\prime}\left[\Pi_{1}\left(Q^{*}, 0\right)\right]-U^{\prime}\left[\Pi_{0}\left(Q^{*}, 0\right)\right]>0$ and thus $\alpha^{*}>0$. Since $\Pi_{0}\left(Q^{*}, 1\right)=\Pi_{1}\left(Q^{*}, 1\right)=(1-$ $p \gamma) P Q^{*}-C\left(Q^{*}\right)$, we have $U^{\prime}\left[\Pi_{1}\left(Q^{*}, 1\right)\right]=U^{\prime}\left[\Pi_{0}\left(Q^{*}, 1\right)\right]$. The producer as such fully insures against the revenue risk by choosing $\alpha^{*}=1$. Eq. (6) with $\beta=0$ and $\alpha^{*}=1$ implies that $Q^{*}=Q_{1}$ that solves $C^{\prime}\left(Q_{1}\right)=(1-p \gamma) P$. Rewrite Eq. (11) as

$$
(1-p \gamma) P-C^{\prime}\left(Q^{\dagger}\right)=(1-p)\left\{1-\frac{U^{\prime}\left[\Pi_{0}\left(Q^{\dagger}, 0\right)\right]}{U^{\prime}\left[\Pi_{1}\left(Q^{\dagger}, 0\right)\right]}\right\}\left[P-C^{\prime}\left(Q^{\dagger}\right)\right]
$$

Since $U^{\prime}\left[\Pi_{0}\left(Q^{\dagger}, 0\right)\right]<U^{\prime}\left[\Pi_{1}\left(Q^{\dagger}, 0\right)\right]$ given risk aversion, it follows from Eq. (A.7) and $C^{\prime \prime}(Q)>0$ that $Q^{\dagger}<Q_{1}$ for all $p \in(0,1)$.

Proof of Proposition 4. Condition (7) and Eq. (14) implies that $\alpha^{*}=0$ if $H(p) \leq 0$. Differentiating Eq. (14) with respect to $p$ yields

$$
\begin{aligned}
H^{\prime}(p)= & -\beta G^{\prime \prime}\left\{U\left(\Pi_{1}^{\max }\right)-U\left[\Pi_{1}\left(Q^{\circ}, 0\right)\right]\right\} U^{\prime}\left[\Pi_{1}\left(Q^{\circ}, 0\right)\right] U^{\prime}\left(\Pi_{1}^{\max }\right) \gamma P Q_{1} \\
& +\left\{\left\{1+\beta G^{\prime}\left\{U\left(\Pi_{1}^{\max }\right)-U\left[\Pi_{1}\left(Q^{\circ}, 0\right)\right]\right\}\right\} U^{\prime \prime}\left[\Pi_{1}\left(Q^{\circ}, 0\right)\right]\left[(1-\gamma) P-C^{\prime}\left(Q^{\circ}\right)\right]\right. \\
& -\beta G^{\prime \prime}\left\{U\left(\Pi_{1}^{\max }\right)-U\left[\Pi_{1}\left(Q^{\circ}, 0\right)\right]\right\} U^{\prime}\left[\Pi_{1}\left(Q^{\circ}, 0\right)\right]^{2}\left[(1-\gamma) P-C^{\prime}\left(Q^{\circ}\right)\right] \\
& -\left\{1+\beta G^{\prime}\left\{U\left(\Pi_{0}^{\max }\right)-U\left[\Pi_{0}\left(Q^{\circ}, 0\right)\right]\right\}\right\} U^{\prime \prime}\left[\Pi_{0}\left(Q^{\circ}, 0\right)\right]\left[P-C^{\prime}\left(Q^{\circ}\right)\right] \\
& \left.+\beta G^{\prime \prime}\left\{U\left(\Pi_{0}^{\max }\right)-U\left[\Pi_{0}\left(Q^{\circ}, 0\right)\right]\right\} U^{\prime}\left[\Pi_{0}\left(Q^{\circ}, 0\right)\right]^{2}\left[P-C^{\prime}\left(Q^{\circ}\right)\right]\right\} \frac{\mathrm{d} Q^{\circ}}{\mathrm{d} p},
\end{aligned}
$$

where $Q_{1}$ solves $C^{\prime}\left(Q_{1}\right)=(1-p \gamma) P$. Since $G^{\prime \prime}(\cdot)>0, U^{\prime \prime}(\Pi)<0$, and $(1-\gamma) P<C^{\prime}\left(Q^{\circ}\right)<$ $P$, Eq. (A.8) implies that $H^{\prime}(p)<0$ if $\mathrm{d} Q^{\circ} / \mathrm{d} p<0$. Totally differentiating Eq. (10) with respect to $p$ yields

$$
\begin{aligned}
& \frac{\mathrm{d} Q^{\circ}}{\mathrm{d} p}=\frac{1}{S O C^{\circ}}\left\{\left\{1+\beta G^{\prime}\left\{U\left(\Pi_{0}^{\max }\right)-U\left[\Pi_{0}\left(Q^{\circ}, 0\right)\right]\right\}\right\} U^{\prime}\left[\Pi_{0}\left(Q^{\circ}, 0\right)\right]\left[P-C^{\prime}\left(Q^{\circ}\right)\right]\right. \\
& \left.-\left\{1+\beta G^{\prime}\left\{U\left(\Pi_{2}^{\max }\right)-U\left[\Pi_{1}\left(Q^{\circ}, 0\right)\right]\right\}\right\} U^{\prime}\left[\Pi_{1}\left(Q^{\circ}, 0\right)\right]\left[(1-\gamma) P-C^{\prime}\left(Q^{\circ}\right)\right]\right\}
\end{aligned}
$$




$$
=\frac{1}{p S O C^{\circ}}\left\{1+\beta G^{\prime}\left\{U\left(\Pi_{0}^{\max }\right)-U\left[\Pi_{0}\left(Q^{\circ}, 0\right)\right]\right\}\right\} U^{\prime}\left[\Pi_{0}\left(Q^{\circ}, 0\right)\right]\left[P-C^{\prime}\left(Q^{\circ}\right)\right]<0,
$$

where the second equality follows from Eq. (10), and the inequality follows from Eq. (A.1) and $C^{\prime}\left(Q^{\circ}\right)<P$. Hence, Eqs. (A.8) and (A.9) imply that $H^{\prime}(p)>0$.

When $p$ approaches zero, $Q^{\circ}$ approaches $Q_{0}$. Hence, we have

$$
\begin{aligned}
H(0)= & \left\{1+\beta G^{\prime}\left\{U\left(\Pi_{1}^{\max }\right)-U\left[\Pi_{1}\left(Q_{0}, 0\right)\right]\right\}\right\} U^{\prime}\left[\Pi_{1}\left(Q_{0}, 0\right)\right] \\
& -\left[1+\beta G^{\prime}(0)\right] U^{\prime}\left[\Pi_{0}\left(Q_{0}, 0\right)\right] .
\end{aligned}
$$

Since $G^{\prime \prime}(\cdot)>0$ and $U^{\prime}\left[\Pi_{1}\left(Q_{2}, 0\right)\right]>U^{\prime}\left[\Pi_{0}\left(Q_{2}, 0\right)\right]$, Eq. (A.10) implies that $H(0)>0$. When $p$ approaches one, both $Q^{\circ}$ and $Q_{1}$ approach $Q_{2}$. Hence, we have

$$
\begin{aligned}
H(1)= & {\left[1+\beta G^{\prime}(0)\right] U^{\prime}\left[\Pi_{1}\left(Q_{2}, 0\right)\right] } \\
& -\left\{1+\beta G^{\prime}\left\{U\left(\Pi_{0}^{\max }\right)-U\left[\Pi_{0}\left(Q_{2}, 0\right)\right]\right\}\right\} U^{\prime}\left[\Pi_{0}\left(Q_{2}, 0\right)\right]
\end{aligned}
$$

It follows from condition (15) and Eq. (A.11) that $H(1)<0$. Since $H^{\prime}(p)<0$, there must exist a unique point, $p^{*} \in(0,1)$, that solves $H\left(p^{*}\right)=0$.

For all $p \in\left(0, p^{*}\right)$, we have $H(p)>0$ so that $\alpha^{*}>0$. In this case, condition (7) holds with equality. Substituting this equation into Eq. (6) yields

$$
\left\{1+\beta G^{\prime}\left\{U\left(\Pi_{1}^{\max }\right)-U\left[\Pi_{1}\left(Q^{*}, \alpha^{*}\right)\right]\right\}\right\} U^{\prime}\left[\Pi_{1}\left(Q^{*}, \alpha^{*}\right)\right]\left[(1-p \gamma) P-C^{\prime}\left(Q^{*}\right)\right]=0 .
$$

Hence, Eq. (A.12) implies that $Q^{*}=Q_{1}$ that solves $C^{\prime}\left(Q_{1}\right)=(1-p \gamma) P$. Evaluating the left-hand side of Eq. (16) at $\alpha^{*}=1$ yields

$$
\beta\left\{G^{\prime}(0)-G^{\prime}\left\{U\left(\Pi_{0}^{\max }\right)-U\left[\Pi_{0}\left(Q_{1}, 1\right)\right]\right\}\right\} U^{\prime}\left[\Pi_{1}\left(Q_{1}, 1\right)\right]<0,
$$

where we have used the fact that $\Pi_{0}\left(Q_{1}, 1\right)=\Pi_{1}\left(Q_{1}, 1\right)=(1-p \gamma) P Q_{1}-C\left(Q_{1}\right)$, and the inequality follows from $G^{\prime \prime}(\cdot)>0$. Hence, we conclude that the optimal coinsurance rate, 
$\alpha^{*} \in(0,1)$, solves Eq. (16). For all $p \in\left[p^{*}, 1\right)$, we have $H(p) \leq 0$ so that $\alpha^{*}=0$. In this case, the producer's optimal output level is $Q^{\circ}$ that solves Eq. (10).

If condition (15) does not hold, Eq. (A.11) implies that $H(1) \geq 0$. Hence, we have $H(p)>0$ for all $p \in(0,1)$. In this case, $\alpha^{*} \in(0,1)$ solves Eq. (16) and the optimal output level is $Q_{1}$ for all $p \in(0,1)$.

Proof of Proposition 5. Rewrite Eq. (10) as

$$
\begin{aligned}
& (1-p \gamma) P-C^{\prime}\left(Q^{\circ}\right)=(1-p)\left[P-C^{\prime}\left(Q^{\circ}\right)\right] \\
& \times\left\{1-\frac{\left\{1+\beta G^{\prime}\left\{U\left(\Pi_{0}^{\max }\right)-U\left[\Pi_{0}\left(Q^{\circ}, 0\right)\right]\right\}\right\} U^{\prime}\left[\Pi_{0}\left(Q^{\circ}, 0\right)\right]}{\left\{1+\beta G^{\prime}\left\{U\left(\Pi_{2}^{\max }\right)-U\left[\Pi_{1}\left(Q^{\circ}, 0\right)\right]\right\}\right\} U^{\prime}\left[\Pi_{1}\left(Q^{\circ}, 0\right)\right]} .\right.
\end{aligned}
$$

Eqs. (20) and (A.14) imply that $Q_{1}<(>) Q^{\circ}$ if $K(p)<(>) 0$. Differentiating Eq. (20) with respect to $p$ yields

$$
\begin{aligned}
K^{\prime}(p)= & \left\{\left\{1+\beta G^{\prime}\left\{U\left(\Pi_{2}^{\max }\right)-U\left[\Pi_{1}\left(Q^{\circ}, 0\right)\right]\right\}\right\} U^{\prime \prime}\left[\Pi_{1}\left(Q^{\circ}, 0\right)\right]\left[(1-\gamma) P-C^{\prime}\left(Q^{\circ}\right)\right]\right. \\
& -\beta G^{\prime \prime}\left\{U\left(\Pi_{2}^{\max }\right)-U\left[\Pi_{1}\left(Q^{\circ}, 0\right)\right]\right\} U^{\prime}\left[\Pi_{1}\left(Q^{\circ}, 0\right)\right]^{2}\left[(1-\gamma) P-C^{\prime}\left(Q^{\circ}\right)\right] \\
& -\left\{1+\beta G^{\prime}\left\{U\left(\Pi_{0}^{\max }\right)-U\left[\Pi_{0}\left(Q^{\circ}, 0\right)\right]\right\}\right\} U^{\prime \prime}\left[\Pi_{0}\left(Q^{\circ}, 0\right)\right]\left[P-C^{\prime}\left(Q^{\circ}\right)\right] \\
& \left.+\beta G^{\prime \prime}\left\{U\left(\Pi_{0}^{\max }\right)-U\left[\Pi_{0}\left(Q^{\circ}, 0\right)\right]\right\} U^{\prime}\left[\Pi_{0}\left(Q^{\circ}, 0\right)\right]^{2}\left[P-C^{\prime}\left(Q^{\circ}\right)\right]\right\} \frac{\mathrm{d} Q^{\circ}}{\mathrm{d} p}
\end{aligned}
$$

Since $G^{\prime \prime}(\cdot)>0, U^{\prime \prime}(\Pi)<0,(1-\gamma) P<C^{\prime}\left(Q^{\circ}\right)<P$, and $\mathrm{d} Q^{\circ} / \mathrm{d} p<0$, it follows from Eq. (A.15) that $K^{\prime}(p)<0$.

When $p$ approaches zero, $Q^{\circ}$ approaches $Q_{0}$. Hence, we have

$$
\begin{aligned}
K(0)= & \left\{1+\beta G^{\prime}\left\{U\left(\Pi_{2}^{\max }\right)-U\left[\Pi_{1}\left(Q_{0}, 0\right)\right]\right\}\right\} U^{\prime}\left[\Pi_{1}\left(Q_{0}, 0\right)\right] \\
& -\left[1+\beta G^{\prime}(0)\right] U^{\prime}\left[\Pi_{0}\left(Q_{0}, 0\right)\right] .
\end{aligned}
$$


Since $G^{\prime \prime}(\cdot)>0$ and $U^{\prime}\left[\Pi_{1}\left(Q_{2}, 0\right)\right]>U^{\prime}\left[\Pi_{0}\left(Q_{2}, 0\right)\right]$, Eqs. (A.10) and (A.16) imply that $H(0)>K(0)>0$. When $p$ approaches one, $Q^{\circ}$ approaches $Q_{2}$. Hence, we have

$$
\begin{aligned}
K(1)= & {\left[1+\beta G^{\prime}(0)\right] U^{\prime}\left[\Pi_{1}\left(Q_{2}, 0\right)\right] } \\
& -\left\{1+\beta G^{\prime}\left\{U\left(\Pi_{0}^{\max }\right)-U\left[\Pi_{0}\left(Q_{2}, 0\right)\right]\right\}\right\} U^{\prime}\left[\Pi_{0}\left(Q_{2}, 0\right)\right] .
\end{aligned}
$$

It follows from condition (15) and Eqs. (A.11) and (A.17) that $H(1)=K(1)<0$. Since $K^{\prime}(p)<0$, there must exist a unique point, $p^{* *} \in\left(0, p^{*}\right)$, at which $K\left(p^{* *}\right)=0$. Since $H(p)>K(p)$ for all $p \in(0,1)$, we must have $p^{* *}<p^{*}$. For all $p \in\left(0, p^{*}\right)$, the producer purchases the coinsurance contract. If $p \in\left(0, p^{* *}\right)$, we have $K(p)>0$ so that $Q_{1}>Q^{\circ}$. If $p \in\left(p^{* *}, p^{*}\right)$, we have $K(p)<0$ so that $Q_{1}<Q^{\circ}$. If $p=p^{* *}$, then $Q_{1}=Q^{\circ}$.

If condition (15) does not hold, Eqs. (A.11) and (A.17) imply that $H(1)=K(1) \geq 0$. Hence, we have $H(p)>0$ and $K(p)>0$ for all $p \in(0,1)$. In this case, $\alpha^{*} \in(0,1)$ and $Q_{1}>Q^{\circ}$ for all $p \in(0,1)$.

\section{References}

Bell, D. E., 1982. Regret in decision making under uncertainty. Operations Research 30, 961-981.

Bell, D. E., 1983. Risk premiums for decision regret. Management Science 29, 1156-1166.

Braun, M., Muermann, A., 2004. The impact of regret on the demand for insurance. Journal of Risk and Insurance 71, 737-767.

Ford, J. L., Mpuku, H. C., Pattanaik, P. K., 1996. Revenue risks, insurance, and the behavior of competitive firms. Journal of Economics 64, 233-246.

Hart, C. E., Babcock, B. A., Hayes, D. J., 2001. Livestock revenue insurance. Journal of Futures Markets 21, 553-580. 
Hau, A., 2006. Production under uncertainty with insurance or hedging. Insurance: Mathematics and Economics 38, 347-359.

Loomes, G., 1988. Further evidence of the impact of regret and disappointment in choice under uncertainty. Economica 55, 47-62.

Loomes, G., Starmer, C., Sugden, R., 1992. Are preferences monotonic-Testing some predictions of regret theory. Economica 59, 17-33.

Loomes, G., Sugden, R., 1982. Regret theory: An alternative theory of rational choice under uncertainty. Economic Journal 92, 805-824.

Loomes, G., Sugden, R., 1987. Testing for regret and disappointment in choice under uncertainty. Economic Journal 97, 118-129.

Machnes, Y., 1995. Deductible insurance and production. Insurance: Mathematics and Economics 17, 119-123.

Machnes, Y., Wong, K. P., 2003. A note on deductible insurance and production, Geneva Papers on Risk and Insurance Theory 28, 73-80.

Mahul, O., Vermersch, D., 2000. Hedging crop risk with yield insurance futures and options. European Review of Agricultural Economics 27, 109-126.

Quiggin, J., 1994. Regret theory with general choice sets. Journal of Risk and Uncertainty $8,153-165$.

Starmer, C., Sugden, R., 1993. Testing for juxtaposition and event-splitting effects. Journal of Risk and Uncertainty 6, 235-254.

Sugden, R., 1993. An axiomatic foundation of regret. Journal of Economic Theory 60, $159-180$.

Wong, K. P., 2000. Insurance and the behavior of competitive firms under revenue risks: A note. Journal of Economics 71, 305-314.

Wong, K. P., 2011. Regret theory and the banking firm: The optimal bank interest margin. Economic Modelling 28, 2483-2487. 